\title{
Evaluation of the impact of rehospitalization in the management of hepatic encephalopathy
}

This article was published in the following Dove Press journal:

International Journal of General Medicine

5 May 2015

Number of times this article has been viewed

\section{Sammy Saab}

Department of Medicine, University of California, Los Angeles,

Los Angeles, CA, USA
Correspondence: Sammy Saab Departments of Medicine and Surgery, University of California, Los Angeles, 200 UCLA Medical Plaza, Suite 2I4,

Los Angeles, CA 90095, USA

Tel +I 3102066705

Fax + I 3102064197

Email ssaab@mednet.ucla.edu
Background: Overt hepatic encephalopathy (HE), which is associated with neuropsychiatric symptoms and neuromuscular dysfunction in patients with liver cirrhosis, is often managed in the hospital setting. Approximately $60 \%$ of eligible patients do not receive prophylactic therapy after an overt HE episode.

Objective: The aim of this review is to evaluate the impact of rehospitalization on costs and clinical outcomes in HE.

Methods: A PubMed search of English-language articles through July 9, 2014 was conducted, and bibliographies of identified publications were reviewed. Abstracts from relevant professional society meetings from 2010 to 2014 were searched. The selected references and abstracts reported on the prevalence, costs, or clinical consequences of rehospitalization in adults with HE.

Data synthesis: HE is a key reason for readmission among patients hospitalized for complications of cirrhosis. Almost $40 \%$ of patients previously hospitalized for HE may be readmitted within 1 year for HE-related reasons. Furthermore, in-hospital US mortality for patients admitted for $\mathrm{HE}$ is about $7 \%$ to $15 \%$. Recurrent $\mathrm{HE}$ and hospitalization for cirrhosis complications are associated with impaired quality of life. In addition, recurrences (especially those requiring hospitalization) may contribute to persistent cognitive deficits (eg, impairments in reaction time, attention, and working memory) after resolution of an acute episode of overt HE.

Conclusion: The economic and clinical consequences of rehospitalization for patients with overt HE underscore the importance of secondary prevention and highlight the need to identify reasons for the undertreatment of patients after hospitalization for overt HE.

Keywords: hospitalization, lactulose, prevention, rifaximin, cirrhosis, cognitive impairment

\section{Introduction}

Hepatic encephalopathy (HE) is a potentially reversible condition associated with neuropsychiatric symptoms and neuromuscular dysfunction of varying severity. ${ }^{1}$ $\mathrm{HE}$ is caused by liver insufficiency, portosystemic shunting, or both. ${ }^{2} \mathrm{HE}$ comprises a spectrum of abnormalities spanning a range from minimal (covert) HE (eg, subtle alterations in cognitive function determined via neuropsychometric tests) to overt $\mathrm{HE}$ (eg, evaluable clinical symptoms, such as generalized motor dysfunction with alterations in consciousness). ${ }^{2,3}$ Additionally, as the condition progresses from minimal HE to overt HE, patients and caregivers may report personality changes, such as apathy, lack of inhibition, and irritability. ${ }^{2}$ Sleep disturbances (eg, reversal of the sleep-wake cycle) and changes in motor function (eg, development of asterixis) and consciousness may also be observed.

$\mathrm{HE}$ is a common complication of decompensated cirrhosis. ${ }^{3} \mathrm{Up}$ to $40 \%$ of patients with cirrhosis eventually develop overt HE, and many patients experience repeated 
episodes (ie, recurrence). ${ }^{2,4}$ The presence of overt HE at the time of an initial diagnosis of cirrhosis has been reported in $10 \%$ to $14 \%$ of patients overall, in $16 \%$ to $21 \%$ of patients diagnosed with decompensated cirrhosis, and in $10 \%$ to $50 \%$ of patients with a transjugular intrahepatic portosystemic shunt. ${ }^{2}$ The prognosis after developing overt HE is poor, with a 1-year survival rate as high as $40 \%$ to $50 \%$ and a 3 -year survival rate of approximately $20 \% 0^{5,6} \mathrm{In}$ a study of US adults on the United Network for Organ Sharing and Organ Procurement and Transplantation Network registry waitlist for liver transplantation, patients with severe (grade 3-4) overt HE at the time of waitlist registration had a significantly greater risk of 90-day mortality than patients without HE (hazard ratio, 1.6; $95 \%$ confidence interval, $1.4,1.9 ; P<0.001){ }^{7}$

HE can progress quickly, and the severity of symptoms often leads to patients being hospitalized for treatment. ${ }^{3}$ In the 2006 Nationwide Inpatient Sample, which included 65,072 discharge records involving hospitalizations due to complications of cirrhosis, $41 \%$ of cirrhosis-related hospitalizations requiring critical care were associated with $\mathrm{HE} .{ }^{8}$ The number of hospital discharges associated with HE based on codes from the International Classification of Diseases, Ninth Revision, Clinical Modification has progressively increased since 2004 and exceeded 400,000 in 2010. ${ }^{9,10}$ Based on the Nationwide Inpatient Sample, the estimated hospitalization incidence for patients with $\mathrm{HE}$ as a primary or secondary diagnosis ranged from 102,293 in 2005 to 115,814 in 2009; the prevalence was $0.33 \%$ of all hospitalizations. ${ }^{11}$ Although hospitalizations related to HE have been increasing, for patients with a primary diagnosis of HE, the mean length of stay decreased between 1993 and 1996 in the USA and has subsequently remained constant at approximately 6 days. ${ }^{9,12-14}$ On average, the length of stay included 0.9 days in the intensive care unit, based on an analysis of a large US hospital database (June 2010 to December 2011). ${ }^{14}$

Although the burden and cost of initial hospitalization for HE and other factors such as treatment for HE generally have been characterized, it is not apparent that the risks and costs associated with rehospitalization have been studied to the same extent. Indeed, the risk of rehospitalization for patients with cirrhosis appears to be substantial. In a retrospective study of patients with decompensated cirrhosis, 69\% (276) of 402 records showed $>1$ readmission related to complications, with a median time to first readmission of 67 days. ${ }^{15}$ The authors reported a median number of readmissions of two (range, 0-40); however, readmissions could have resulted from any of the reported complications of cirrhosis and were not limited to HE.
The objective of this systematic review is to evaluate the impact of rehospitalization on costs and clinical outcomes for patients with cirrhosis initially hospitalized for HE.

\section{Methods}

A systematic review of the PubMed medical literature was conducted for English-language articles with no time limitation (up to February 18, 2015). The primary search was performed using the keywords "hepatic encephalopathy", "hospitalization", "admission", "rehospitalization", and "readmission". To ensure all relevant articles were identified, additional (secondary) search terms and search strings were tested with the following keywords: "quality of life", "factor", "predictors", "mortality", "survival", "transplantation", "burden", "cost", "no treatment", "untreated", "placebo", and "prophylaxis". Selection criteria (papers discussing randomized, controlled trials; observational studies; and meta-analyses) were applied to the initial searches to enrich the quality of the studies reported. These criteria were later relaxed to expand the pool of eligible studies to include openlabel, retrospective, uncontrolled, and cross-sectional study designs. Bibliographies of identified publications were also reviewed for relevant references that had escaped detection using the predefined search terms.

Because the initial literature search identified a relatively low number of studies, the review was expanded to include abstracts published for pertinent medical conferences between 2010 and 2014. Included were abstracts for the American College of Gastroenterology annual meeting, Digestive Disease Week, The Liver Meeting ${ }^{\circledR}$ (American Association for the Study of Liver Diseases), and The International Liver Congress ${ }^{\mathrm{TM}}$ (European Association of the Study of the Liver), based on a search for "encephalopathy". The search of medical conferences was limited to those taking place in the last 5 years at the time the initial searches were conducted; the author anticipated a larger number of studies would be identified in the PubMed literature. All references and abstracts selected for inclusion reported on the prevalence, costs, or clinical consequences of rehospitalization in adult patients with HE.

\section{Results}

Outcomes of the systematic search are summarized in the PRISMA (Preferred Reporting Items for Systematic Reviews and Meta-Analyses) flow diagram (Figure 1). Results of the PubMed search yielded 95 studies; an additional 27 studies were identified from medical conferences $(n=9)$ and secondary search terms $(n=18)$. Studies from the 


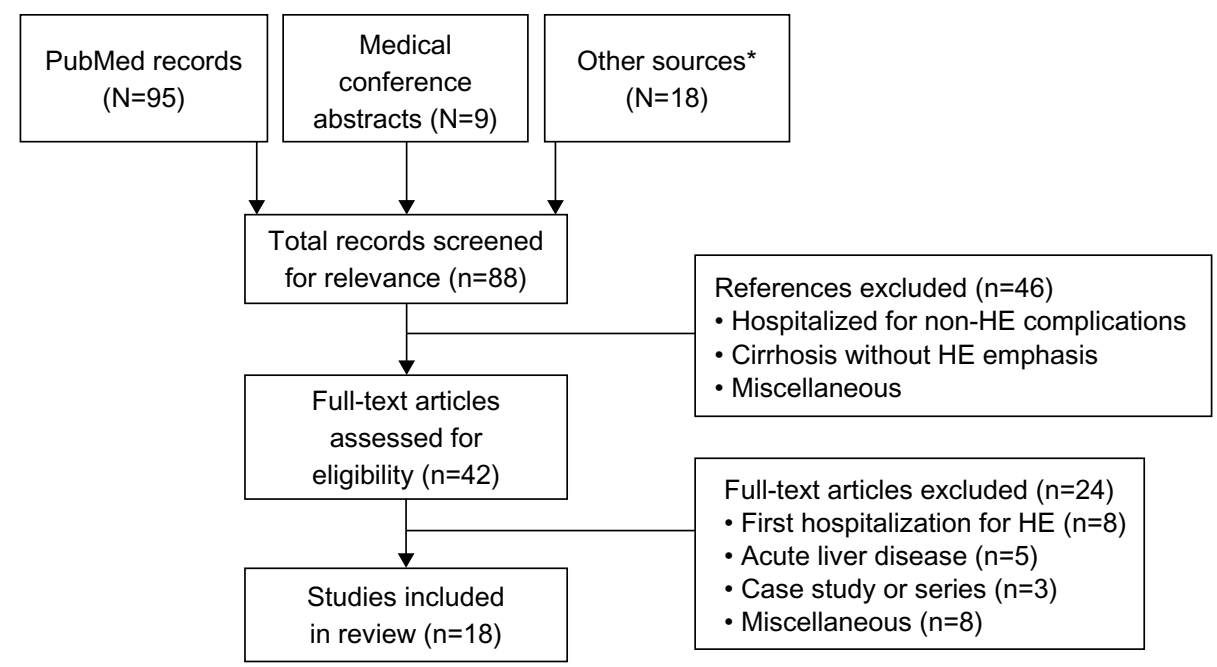

Figure I PRISMA (Preferred Reporting Items for Systematic Reviews and Meta-Analyses) flow diagram summary of search outcomes. Data from systematic review of PubMed database performed initially in July 2014 and again in February 2015.

Note: *Secondary search strings.

Abbreviation: $\mathrm{HE}$, hepatic encephalopathy.

latter category included pertinent data for inclusion in the review that were not identified when the primary search string was performed. Of those studies initially identified, the majority $(\mathrm{n}=92)$ were excluded upon further review. The primary reasons for exclusion were studies in animals, hospitalization attributed to other complications of cirrhosis, acute liver disease, and initial hospitalization event without recurrence, among others. This review includes results from 12 journal publications and six abstracts (Table 1). ${ }^{6,8,9,11,12,14,16-27}$

\section{Readmission rates in patients with $\mathrm{HE}$}

A retrospective analysis of data from $>500$ US hospitals assessed hospital readmissions in 8,766 adults discharged from the hospital between June 2010 and December 2011 with a primary diagnosis of HE. ${ }^{14}$ Overall, $17.6 \%$ of discharged patients were readmitted within 30 days, and $39.5 \%$ were readmitted within 1 year for HE-related reasons. Incidence rates for all-cause readmission were $27.4 \%$ at 30 days and $56.4 \%$ at 1 year. In another retrospective US study, approximately one-third of 402 patients with decompensated cirrhosis had HE at admission, 78\% (314 of 402) of patients were readmitted during a median follow-up period of 203 days, with $88 \%$ ( 276 of 314 ) of patients having $\geq 1$ non-elective readmission. ${ }^{22}$ The median time to first readmission was 67 days, with $14 \%$ of patients readmitted within 1 week and $37 \%$ within 1 month. Overall, $22 \%$ of readmissions within 1 month were considered preventable. In fact, recurrent HE was among the most preventable reasons for readmission. Notably, after adjusting for potential confounding factors, patients with more frequent readmissions had a significantly increased risk of mortality.

Furthermore, a retrospective review analyzed data from 139 patients admitted to a US tertiary hospital for the treatment of complications of liver cirrhosis and reported that HE was an indication for admission in $36 \%$ of these patients. ${ }^{24,25}$ Of the $31 \%$ of patients readmitted within 1 month, $47 \%$ were readmitted for HE. In an attempt to analyze possible causes of readmission due to $\mathrm{HE}$, an analysis of medical records of 145 patients admitted with overt HE to a single hospital identified poor social support, failure to fill a prescription, and lack of follow-up with a health care provider as factors associated with a high likelihood of readmission. ${ }^{26}$

\section{Costs of hospitalization}

Hospitalization for HE is associated with substantial economic burden. The total national cost of hospitalizations of patients with HE (primary or secondary diagnosis) increased from US $\$ 1.7$ billion in 2005 to US $\$ 2.0$ billion in $2009 .{ }^{11}$ The total cost per HE-related hospital discharge increased from US $\$ 22,511$ in 2004 to US\$37,598 in $2010 .^{9}$ This analysis included patients with any International Classification of Diseases, Ninth Revision, Clinical Modification discharge diagnosis code indicative of HE (ie, not limited to patients with a primary or secondary diagnosis of HE). The mean hospital cost for HE-related hospitalization (primary or secondary diagnosis) did increase between 2005 (US\$16,512) and 2009 (US\$17,812), but the difference was not statistically significant. ${ }^{11}$ A study reported that the mean hospital cost in 2003 for patients admitted with a primary diagnosis of 


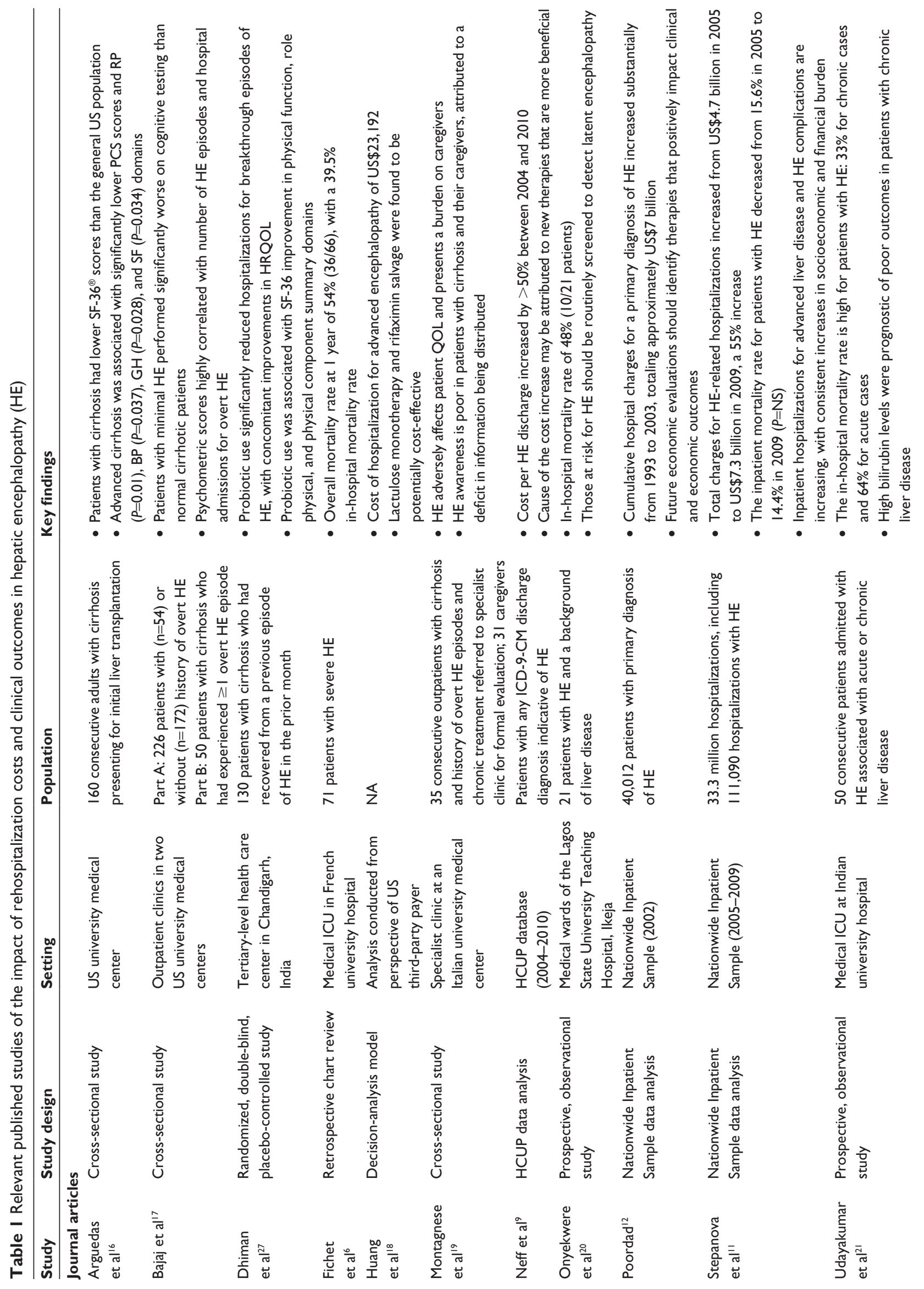



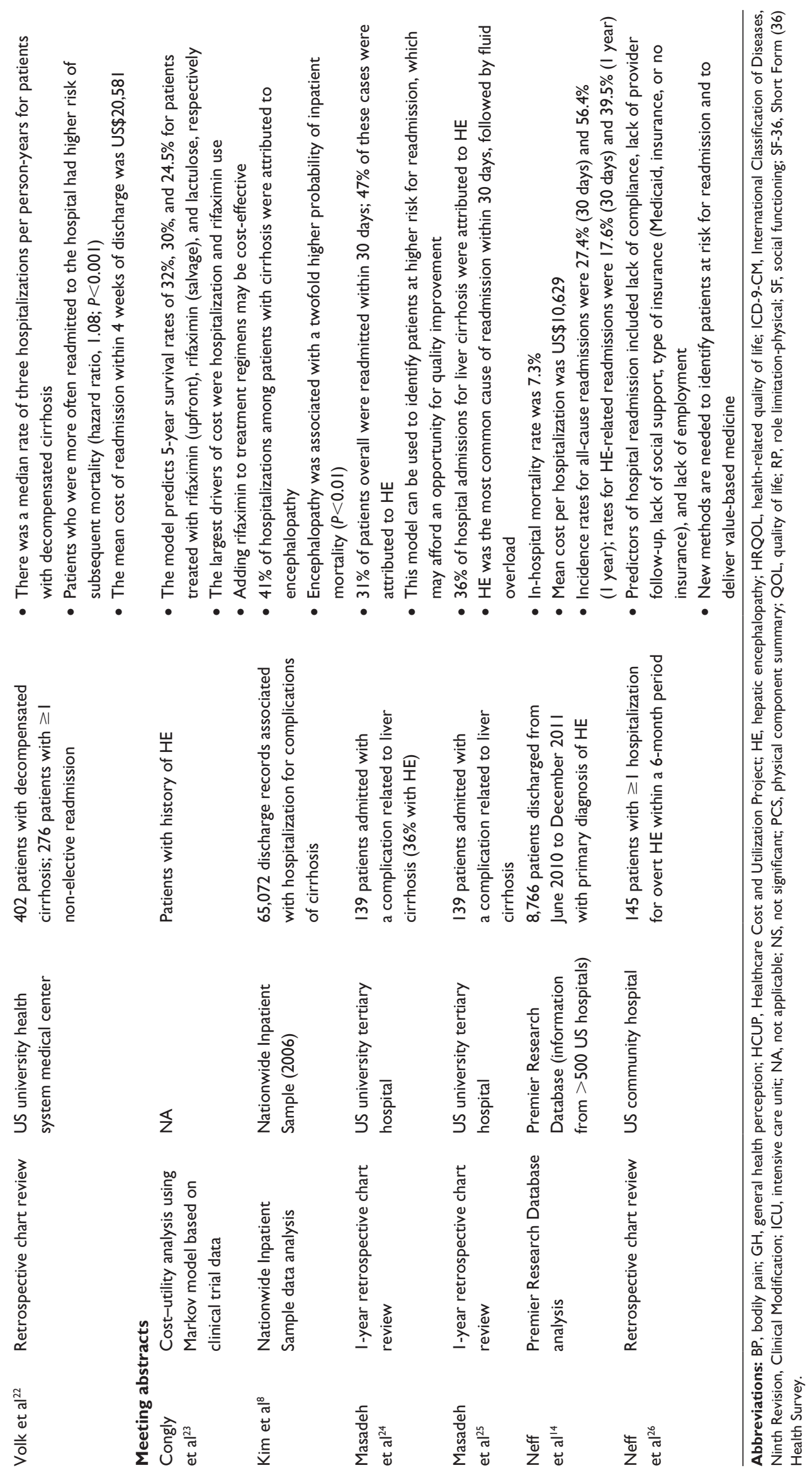
HE was US $\$ 23,192 .{ }^{12}$ The mean cost of hospitalization for HE (as a primary diagnosis) in a large ( $>500)$ US hospital database (June 2010 to December 2011) was US $\$ 10,629$, with room and board accounting for approximately $50 \%$ of overall hospitalization costs (mean, US\$5,518) and pharmacy costs accounting for $11.5 \%$ of overall hospitalization costs (mean, US\$1,223). ${ }^{14}$

In a study of a university health system, the mean cost of readmission for patients with decompensated cirrhosis was US $\$ 25,898$ for readmission within 1 week and US\$20,581 for readmission between weeks 1 and $4 .^{22}$

Overall, these data underscore the high costs of hospitalization for $\mathrm{HE}$ and the economic impact of rehospitalization.

\section{In-hospital mortality rates}

For patients hospitalized with cirrhosis, HE doubles the risk of in-hospital mortality. ${ }^{8}$ In US hospitals, the in-hospital mortality rate for overt $\mathrm{HE}$ ranges between approximately $7 \%$ and $15 \% .{ }^{11,14}$ Higher in-hospital mortality rates have been reported in patients with severe $\mathrm{HE}(\sim 40 \%)^{6}$ and in patients treated in developing countries $(\sim 33 \%$ to $50 \%)$. ${ }^{20,21}$ Risk factors for inpatient mortality in patients with HE include a higher number of concomitant diagnoses, comorbid fluid and electrolyte disorders, more procedures during admission, and major or extreme severity of illness. ${ }^{11}$ Interestingly, a 2014 study reported that non-hospitalized patients with cirrhosis and minimal HE were at an increased risk of hospitalization compared with patients without minimal HE, when adjusted for sex, age, and cirrhosis etiology (hazard ratio, 2.5; 95\% confidence interval, $1.4,4.5 ; P=0.002){ }^{28}$

\section{Impact on health-related quality of life}

Cirrhosis and overt HE are both associated with impairment in health-related quality of life (QOL). In patients with cirrhosis and varying degrees of neuropsychiatric impairment, the severity of HE was significantly associated with patient QOL scores as well as the degree of caregiver burden. ${ }^{19}$ Among 160 consecutive adults with cirrhosis presenting for an initial evaluation for liver transplantation in a university setting between January 2001 and September 2001, 125 patients (78\%) had HE (36 with minimal HE and 89 with overt HE).${ }^{16}$ Compared with the general US population, patients with cirrhosis had poorer health-related QOL based on Short Form (36) Health Survey (SF-36 ${ }^{\circledR}$ ) domain and summary scores. Patients with cirrhosis and overt HE experienced significantly worse physical and mental healthrelated QOL than those without HE. In addition, the reduction in health-related QOL related to HE was independent of the
Child-Pugh score. Of note, the physical component summary score of the SF-36 was lower in patients with cirrhosis who had previously been hospitalized than in patients who had never been hospitalized. This observation may be related to an increase in disease severity among patients requiring hospitalization and may also be related to hospitalizations for complications of cirrhotic decompensation, such as HE.

\section{Impact on cognitive function and residual cognitive impairment}

Unfortunately, cognitive deficits have been shown to persist after an episode of overt HE resolves. In a cross-sectional study of 226 patients with cirrhosis, patients with a history of overt HE ( $\mathrm{n}=54)$ performed significantly worse than patients without a history of overt HE $(n=172)$ on psychometric tests. ${ }^{17} \mathrm{~A}$ cross-sectional study assessed cognitive function in 50 patients with cirrhosis who had experienced $\geq 1$ episode of overt HE. Of the 50 patients, 32 were being treated with lactulose monotherapy, eleven were being treated with rifaximin monotherapy, and seven were being treated with rifaximin and lactulose combination therapy. All patients had normal mental status at the time of testing. In that study, the severity of cognitive impairment increased both with the number of episodes of overt HE and with the number of hospitalizations related to overt HE. Furthermore, the number of previous episodes and the number of hospitalizations for overt HE correlated with psychometric scores, which were used to assess severity of residual cognitive impairment (Table 2). ${ }^{17}$

\section{Discussion}

To reduce the risk of HE recurrence, the American Association for the Study of Liver Diseases and European Association of the Study of the Liver recommend prophylactic treatment after an episode of overt HE resolves. ${ }^{2}$ However, routine prophylaxis is not recommended for preventing HE after a transjugular intrahepatic portosystemic shunt. ${ }^{2}$ Medications commonly administered to prevent the recurrence of overt HE are similar to those for treating overt HE episodes and include lactulose and rifaximin - in fact, rifaxi$\mathrm{min}$ is indicated for reducing the risk of overt HE recurrence in adults (Figure 2). ${ }^{2}$ Per treatment guidelines, "[r] ifaximin added to lactulose is the best-documented agent to maintain remission in patients who have already experienced one or more bouts of OHE [overt hepatic encephalopathy] while on lactulose treatment after their initial episode of OHE". ${ }^{2}$ Prophylactic therapy to prevent HE recurrence is usually continued indefinitely, until liver transplantation or death, but 
Table 2 Correlation between psychometric tests and the number of hospitalizations for or episodes of overt hepatic encephalopathy $(\mathrm{HE})(\mathrm{N}=50)$

\begin{tabular}{|c|c|c|c|c|c|}
\hline \multirow[t]{2}{*}{$\overline{\text { Psychometric test }^{a}}$} & \multirow[t]{2}{*}{ Cognitive function(s) evaluated } & \multicolumn{2}{|c|}{$\begin{array}{l}\text { Number of } \\
\text { hospitalizations } \\
\text { for overt HE }\end{array}$} & \multicolumn{2}{|c|}{$\begin{array}{l}\text { Number } \\
\text { of episodes of } \\
\text { overt HE }\end{array}$} \\
\hline & & $r$ & $P$-value & $r$ & $P$-value \\
\hline Digit symbol test (raw score) & Psychomotor speed, attention, and visual memory & -0.39 & 0.02 & -0.46 & 0.009 \\
\hline ICT lures (n) & Response inhibition, attention, and working memory & 0.50 & 0.002 & 0.59 & 0.001 \\
\hline ICT targets (\% correct) & Reaction time & -0.43 & 0.009 & -0.44 & 0.015 \\
\hline Number connection-A (speed) & Psychomotor speed & 0.26 & 0.144 & 0.22 & 0.238 \\
\hline Number connection-B (speed) & $\begin{array}{l}\text { Psychomotor speed, set shifting (ie, mental flexibility } \\
\text { in switching between tasks), and divided attention }\end{array}$ & 0.35 & 0.047 & 0.35 & 0.05 \\
\hline Block design test (raw score) & $\begin{array}{l}\text { Visuomotor coordination, visuospatial reasoning, praxis, } \\
\text { and psychomotor speed }\end{array}$ & -0.11 & 0.631 & -0.20 & 0.378 \\
\hline
\end{tabular}

Notes: Bold values indicate statistical significance. ${ }^{a} \mathrm{~A}$ high score on number connection-A, connection-B, and ICT lures, and a low score on digit symbol, block design, and ICT target number indicate poor psychometric performance.

Adapted with permission from Gastroenterology, vol I38, no 7, Bajaj JS, Schubert CM, Heuman DM, et al. Persistence of cognitive impairment after resolution of overt hepatic encephalopathy, pages 2332-2340, Copyright 2010 with permission from Elsevier. ${ }^{17}$

Abbreviation: ICT, inhibitory control test.

discontinuation may be considered if the precipitating factors have been well controlled or if a patient's liver function or nutritional status has improved. ${ }^{2}$

Despite professional society recommendations for prophylactic therapy, many patients do not appear to be receiving preventive treatment after $\geq 1$ episode of overt HE. An analysis of national claims for medical and hospital activity from 2009 to 2011 indicated that more than $60 \%$ of eligible patients with overt $\mathrm{HE}$ did not receive ongoing prophylactic therapy during each year studied. ${ }^{29}$ The apparent undertreatment of patients after an episode of overt HE may potentially result in preventable HE recurrences and, consequently, preventable rehospitalizations related to these recurrences. The burden and cost implications of undertreatment and potential resultant rehospitalizations for $\mathrm{HE}$ episodes were the subject of this review.

Hospital readmissions after discharge for decompensated cirrhosis, and for HE in particular, are common in patients with cirrhosis. In addition, a considerable number of patients are readmitted within 1 month of hospital discharge, ${ }^{14}$ and rehospitalization for $\mathrm{HE}$ imposes a substantial economic burden. Not surprisingly, statistical modeling data suggest that costs associated with hospitalization are some of the largest contributors to overall costs of HE treatment. ${ }^{23}$ Overall, given the various methodologies applied across studies to determine costs (eg, review of Current Procedural Terminology codes selected), it is difficult to compare results. Indeed, differences exist in the availability and interpretation of

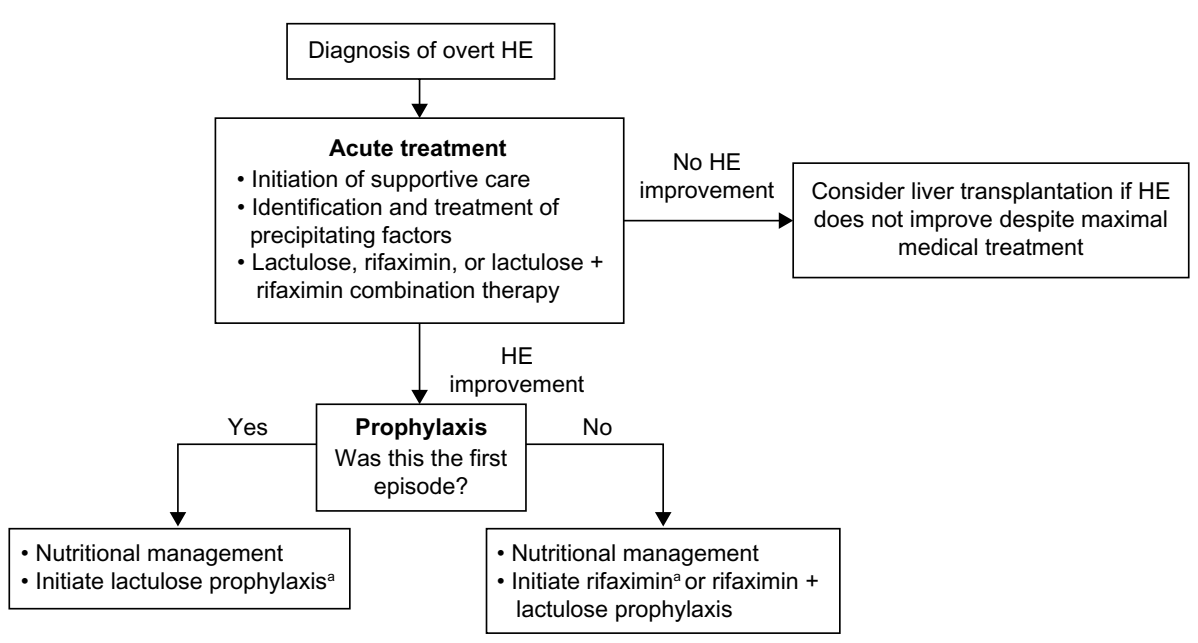

Figure 2 Acute and long-term management of overt hepatic encephalopathy (HE).

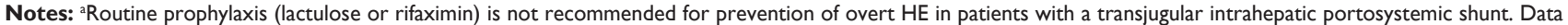
from Vilstrup H, Amodio P, Bajaj J, et al. ${ }^{2}$ 
Current Procedural Terminology codes, studies are conducted across divergent time periods in which health care inflation may vary considerably, studies prospectively enroll or retrospectively review cases of different patient populations with varied severity of disease, and services offered tend to become more complex and costly over time. In the present review, the data support a substantial cost burden associated with hospitalizations related to $\mathrm{HE}$ and suggest that the cost burden has increased over time.

Rehospitalization for HE may lead to adverse consequences for patients with cirrhosis, including increased mortality, impaired health-related QOL, and residual cognitive impairment. Regarding the effects of cognitive impairment, key findings from studies identified for this review support a hypothesis that repeated overt HE episodes may be neurotoxic and may lead to chronic neurologic damage, despite the apparent return of normal mental status initially after treatment. In a study by Bajaj et al, ${ }^{30}$ cognitive impairment was observed across all tests employed, including persistent and cumulative deficits in memory, response inhibition, and learning in patients who had experienced episodes of overt HE. The potential for persistent neurologic damage is an interesting hypothesis that has primarily been reported on in the context of patients observed after liver transplantation. ${ }^{31}$ In one such study, cognitive dysfunction was more pronounced in patients who had experienced overt HE prior to liver transplantation. ${ }^{31}$ Therefore, it will be important in the future to examine the potential for clinical and economic sequelae to extend beyond the primary HE episode.

Additionally, mortality rates remain high for patients with cirrhosis; however, some deaths attributed to an HE episode may be preventable. Notably, guidelines support the importance of prophylactic therapy to help prevent repeated episodes of overt HE in, and the consequent rehospitalization of, patients with cirrhosis.

\section{Limitations}

This review has several important limitations. For instance, most of the studies identified were not prospective but, rather, retrospective chart reviews, smaller cross-sectional studies, or sample surveys. In addition, there was significant heterogeneity in the outcomes measured in the identified studies. Nevertheless, data are accumulating to support the idea that HE-related rehospitalization represents an important unmet medical need. Because the published literature on HE-related rehospitalization is limited, this review included medical conference abstracts, which are considered by some to be less desirable sources of information. In addition, estimated hospitalization costs for HE were used as a proxy for rehospitalization costs in the absence of more specific data. Furthermore, for QOL and cognitive functioning, associations with rehospitalization were reported, but impairments may be related to HE recurrence rather than to rehospitalization per se.

\section{Conclusion}

The economic and clinical consequences of rehospitalization for patients with overt HE underscore the importance of secondary prevention. Nonetheless, about $60 \%$ of patients do not receive ongoing prophylactic therapy to prevent $\mathrm{HE}$ recurrence after discharge. ${ }^{29}$ It is unclear whether the low rate of prophylaxis is the result of patients not filling prescriptions or providers not prescribing prophylactic therapy. Further studies are warranted to help identify the reasons for the undertreatment of patients after hospitalization for overt HE to enable health care providers to better address this gap in medical care.

\section{Acknowledgments}

Technical editorial and medical writing assistance was provided, under the direction of the author, by Larry Nelson, $\mathrm{PhD}$, and Mary Beth Moncrief, PhD, Synchrony Medical Communications, LLC, West Chester, PA, USA. Funding for this support was provided by Salix Pharmaceuticals, Inc., Raleigh, NC, USA.

\section{Disclosure}

Dr Saab serves as a consultant and on the speakers' bureau for and holds stock in Salix Pharmaceuticals, Inc. The author declares no other conflicts of interest in this work.

\section{References}

1. Blei AT, Córdoba J; Practice Parameters Committee of the American College of Gastroenterology. Hepatic encephalopathy. Am J Gastroenterol. 2001;96(7):1968-1976.

2. Vilstrup H, Amodio P, Bajaj J, et al. Hepatic encephalopathy in chronic liver disease: 2014 Practice Guideline by the American Association for the Study of Liver Diseases and the European Association for the Study of the Liver. Hepatology. 2014;60(2):715-735.

3. Leise MD, Poterucha JJ, Kamath PS, Kim WR. Management of hepatic encephalopathy in the hospital. Mayo Clin Proc. 2014;89(2):241-253.

4. Amodio P, Del Piccolo F, Pettenò E, et al. Prevalence and prognostic value of quantified electroencephalogram (EEG) alterations in cirrhotic patients. J Hepatol. 2001;35(1):37-45.

5. Bustamante J, Rimola A, Ventura PJ, et al. Prognostic significance of hepatic encephalopathy in patients with cirrhosis. J Hepatol. 1999;30(5): 890-895.

6. Fichet J, Mercier E, Genée O, et al. Prognosis and 1-year mortality of intensive care unit patients with severe hepatic encephalopathy. J Crit Care. 2009;24(3):364-370. 
7. Wong RJ, Gish RG, Ahmed A. Hepatic encephalopathy is associated with significantly increased mortality among patients awaiting liver transplantation. Liver Transpl. 2014;20(12):1454-1461.

8. Kim WR, Kamath PS, Shah N. Utilization and outcome of critical care in patients with cirrhosis in the US. Hepatology. 2010;52(S1): 910A-911A.

9. Neff GW, Kemmer N, Duncan C, Alsina A. Update on the management of cirrhosis - focus on cost-effective preventative strategies. Clinicoecon Outcomes Res. 2013;5:143-152.

10. Chacko KR, Sigal SH. Update on management of patients with overt hepatic encephalopathy. Hosp Pract (1995). 2013;41(3):48-59.

11. Stepanova M, Mishra A, Venkatesan C, Younossi ZM. In-hospital mortality and economic burden associated with hepatic encephalopathy in the United States from 2005 to 2009. Clin Gastroenterol Hepatol. 2012;10(9):1034-1041.

12. Poordad FF. Review article: the burden of hepatic encephalopathy. Aliment Pharmacol Ther. 2007;25 Suppl 1:3-9.

13. Neff G. Pharmacoeconomics of hepatic encephalopathy. Pharmacotherapy. 2010;30(5 Pt 2):28S-32S.

14. Neff GW, Barrett AC, Graham CM, Gayle J, Ernst FR. Hospital costs, length of stay, and readmission rates in a cohort of cirrhotic patients discharged with hepatic encephalopathy. Hepatology. 2013;58(S1): 390A-391A.

15. Volk ML, Tocco RS, Bazick J, Rakoski MO, Lok AS. Hospital readmissions among patients with decompensated cirrhosis. Am J Gastroenterol. 2012;107(2):247-252.

16. Arguedas MR, DeLawrence TG, McGuire BM. Influence of hepatic encephalopathy on health-related quality of life in patients with cirrhosis. Dig Dis Sci. 2003;48(8):1622-1626.

17. Bajaj JS, Schubert CM, Heuman DM, et al. Persistence of cognitive impairment after resolution of overt hepatic encephalopathy. Gastroenterology. 2010;138(7):2332-2340.

18. Huang E, Esrailian E, Spiegel BM. The cost-effectiveness and budget impact of competing therapies in hepatic encephalopathy - a decision analysis. Aliment Pharmacol Ther. 2007;26(8):1147-1161.

19. Montagnese S, Amato E, Schiff S, et al. A patients' and caregivers' perspective on hepatic encephalopathy. Metab Brain Dis. 2012;27(4): $567-572$.
20. Onyekwere CA, Ogbera AO, Hameed L. Chronic liver disease and hepatic encephalopathy: clinical profile and outcomes. Niger J Clin Pract. 2011;14(2):181-185.

21. Udayakumar N, Subramaniam K, Umashankar L, Verghese J, Jayanthi V. Predictors of mortality in hepatic encephalopathy in acute and chronic liver disease: a preliminary observation. J Clin Gastroenterol. 2007; 41(10):922-926.

22. Volk ML, Tocco RS, Bazick J, Rakoski MO, Lok AS. Hospital readmissions among patients with decompensated cirrhosis. Am J Gastroenterol. 2012;107(2):247-252.

23. Congly SE, Leise MD, Kim WR. Cost effectiveness of rifaximin in the treatment of hepatic encephalopathy. Hepatology. 2013; 58(S1):869A.

24. Masadeh MM, Gonzalez-Fraga JD, Al-afghani AH, et al. Compliance with process of care among patients hospitalized with liver cirrhosis Gastroenterology. 2014;146(5 Suppl 1):S-986.

25. Masadeh MM, Hussain F, Spratt H, Sonstein L, El Haija MA, Soloway RD. A novel model to predict the likelihood of readmission within 30 days in patients hospitalized with liver cirrhosis. Gastroenterology. 2014;146(5 Suppl 1):S-986.

26. Neff GW, Kemmer N, Parkinson E, Fallon EC, Alsina A. Hospital readmissions metrics (HRM) related to hepatic encephalopathy (HE). Hepatology. 2013;58(S1):890A-891A.

27. Dhiman RK, Rana B, Agrawal S, et al. Probiotic VSL\#3 reduces liver disease severity and hospitalization in patients with cirrhosis: a randomized, controlled trial. Gastroenterology. 2014;147(6):1327-1337.

28. Patidar KR, Thacker LR, Wade JB, et al. Covert hepatic encephalopathy is independently associated with poor survival and increased risk of hospitalization. Am J Gastroenterol. 2014;109(11):1757-1763.

29. Neff GW, Frederick RT. Assessing treatment patterns in patients with overt hepatic encephalopathy. Hepatology. 2012;56(Suppl 1):945A.

30. Bajaj JS, Schubert CM, Heuman DM, et al. Persistence of cognitive impairment after resolution of overt hepatic encephalopathy. Gastroenterology. 2010;138(7):2332-2340.

31. Sotil EU, Gottstein J, Ayala E, Randolph C, Blei AT. Impact of preoperative overt hepatic encephalopathy on neurocognitive function after liver transplantation. Liver Transpl. 2009;15(2):184-192.
International Journal of General Medicine

\section{Publish your work in this journal}

The International Journal of General Medicine is an international, peer-reviewed open-access journal that focuses on general and internal medicine, pathogenesis, epidemiology, diagnosis, monitoring and treatment protocols. The journal is characterized by the rapid reporting of reviews, original research and clinical studies across all disease areas.

\section{Dovepress}

A key focus is the elucidation of disease processes and management protocols resulting in improved outcomes for the patient. The manuscript management system is completely online and includes a very quick and fair peer-review system. Visit http://www.dovepress.com/ testimonials.php to read real quotes from published authors. 